\title{
Computer information security management in big data environment
}

\author{
Xie Xiuye \\ LINYI UNIVERSITY, Shandong, Linyi 276400
}

Keywords: Big data; computer information security; management strategy

\begin{abstract}
In the context of the development of big data, science and technology are constantly improving. In order to protect the user's information security, it is necessary to make technical improvements to the computer network, to improve the security configuration of the computer, to improve the user's security awareness, and to prevent the leakage of information security. This paper focuses on the analysis of computer information security management strategies and protection measures in the context of big data environment.
\end{abstract}

\section{Introduction}

With the continuous development of computer networks, the development of today's society is closely related to information. The development of information technology is to facilitate people to quickly obtain information, then to share and transmit information. The development of computer technology is closely related to our lives. However, in the process of rapid information development, people's information security has become a hidden danger. If people's information security cannot be guaranteed, information technology will not be able to meet the needs of social development no matter how it is advanced.

\section{The importance of computer information security management in big data environment}

Computer technology is mainly the process of collecting, processing and storing data. It is widely used in various fields, and its application scope is related to the confidentiality of the country and the privacy protection of individuals. With the rapid development of computer technology, information security has become a major project. Once the information is leaked, it will bring huge losses to individuals and even enterprises. In recent years, the problem of information theft and data leakage has become more and more serious, and it has not been well contained. There are very serious loopholes in economic construction. The scope of information security design is very wide. Once the confidential business information is leaked, the impact on the whole society is very great. If the security protection of the computer system is compromised by the hacker, the loss of information will cause the system to collapse, which will bring great economic losses to the enterprises and the customers. Therefore, strengthening information security management is the stress of modern economic development. Technical improvement of computer networks is the key to ensuring computer information security.

\section{The concept and characteristics of information security in a big data environment}

\subsection{The concept of computer information security}

Computer network is a general term for computers with independent functions and its external devices in different geographical conditions. It mainly uses communication lines to connect them together. Information transfer and sharing are achieved with the help of operating systems, software 
management, and communication protocols. The main device of the Internet is routers. The routers connects the computer to implement the function of transmitting information. While, computer information security refers to the use of related technologies to enable the network to process data properly, and to ensure the security, integrity and confidentiality of data. It is the main function of network information security that it has certain controllability for network information security. Therefore, to ensure the security of computer information, it is necessary to establish a complete security protection system.

\subsection{The characteristics of information security in big data environment}

In the context of the development of the big data era of information explosion, the amount of information of users is very large, and varieties of information are involved, coupled with the rapid spread of information, the security of information is not guaranteed. Therefore, information security issues need to be resolved. The main feature of information in the context of the era of big data is the large amount of storage and fast propagation speed. Encryption technology is required for the transmission process. While performing big data information transmission, it is necessary to securely encrypt the information. Data transmission is generally transmitted or shared in the form of text, images, and video. The efficiency of information processing data directly affects the format of the data. Therefore, the processing of data in the big data environment and the protection of information security need to be technically focused.

\section{The risk of computer information security in big data environment}

\subsection{Natural influence}

In order to realize the communication of data, the computer network needs the support of hardware devices to play its own role. However, these hardware devices are not able to withstand the damage caused by natural disasters for a long time. The network equipment problem caused by natural disasters is an objective condition for information security leakage. When the equipment encounters natural disasters such as rain, snow, earthquakes, etc., the hardware devices connected to the computer will be damaged to varying degrees, and the loss will affect the information security of the computer. For example, in the process of data transmission by a computer, the transmission is interrupted due to the influence of natural factors, so that the information is lost, and no timely backup processing is performed. At this point, it is necessary to have certain quality requirements for the hardware of the computer network equipment, and this can effectively resist data damage caused by natural disasters.

\subsection{The problem of the computer itself}

The important basis for ensuring that the computer's applications are functioning properly is the computer's operating system. The operating system is composed of different software and is the core of computer technology. Different operating systems have very strong management functions, which can effectively manage the running status of the computer hardware and software. Computer operating system is the key to maintaining the development of computer networks. Because the computer network itself has certain defects, the network security problems of the computer sometimes have different levels of changes. However, due to the computer's own problems, the data will be threatened by the network during the process of transmission and storage. And there are many types of security risks in the network, which are unpredictable. There are attacks from hackers, threats from network communication protocols. The main threat is that users who use computers do not have a comprehensive understanding of the use of the network, which makes it 
easy to open connections or arbitrarily enter accounts and passwords during actual operations. This is an important reason for the leakage of network information security. This reason makes the computer network in a very unstable environment, which is not conducive to the development of computer networks.

\subsection{The spread of information security}

In the context of the era of big data network development, information will pass through a very complex network environment in the process of dissemination, and network protocols are used to disseminate data information. Information is likely to be leaked during transmission. Today's network protocol HTTP is a typical protocol for ensuring network secure transmission. However, due to the instability of the network environment, there are many loopholes in the transmission process of the network protocol, which has a certain impact on the security of information transmission. Although the cloud data platform provides storage space for big data, the cloud data platform, as a third-party service platform, also has certain security risks in information security.

\subsection{Information leakage}

With the continuous development of information technology, information technology is widely used in various fields. Its frequency and scope of use are also constantly expanding. Therefore, more and more information is involved in computers. For enterprises, the information in the enterprise is very confidential, which is related to the survival and development of the enterprise, and they need to monitor the information security. However, in real life, some lawless people always use illegal means to invade the database of enterprises, causing information leakage of enterprises. These valuable information leaks are a fatal blow to the company. Not only will it cause economic losses, but even bring instability to the development of the entire society, and generate fear of information security. With the rapid development of computer technology, computer technology has changed people's lives. While bringing some conveniences, huge amounts of information bring certain security risks to people's information security, especially the loopholes in computers. There are some loopholes in the process of using, which affect people's normal use. In addition, many software needs to be registered before it can be used. However, due to the vulnerability of the software in the design process, the user's information leakage may occur, so it is necessary to improve the security risk of the software and protect the user's information security.

\section{Preventive measures for computer information security in big data environment}

\subsection{Overall management of the computer}

Check the hardware and software of the computer thoroughly. First, start the maintenance of the computer from the hardware system. Regularly check the use of the computer's hardware system and maintain the hardware equipment. Replace hardware devices that cannot keep up with the times, and update hardware that is aging or worn out in a timely manner. At the same time, the computer software is used to kill the virus, and the rubbish files in the computer are cleaned in time. At the same time, the data information should be encrypted and protected to prevent the leakage of information caused by virus intrusion and to ensure the safety of user's data. Do a good job in security management and prevention of computer software, update the computer's protection software in a timely manner, and download the latest patches to ensure the normal operation of the computer software system, so that the computer's information security level is higher.

\subsection{Encrypt the information security of computers}

The data in the computer needs technical support. Data encryption technology is a key 
technology in computer network security. Data security issues are most evident in the processing of big data and the transmission of trade secrets. In the current development, data encryption technology is divided into two types: symmetric encryption technology and asymmetric encryption technology. The most widely used is asymmetric data encryption technology. The asymmetric encryption technology is used to encrypt and decrypt information. In the process of encryption and decryption, different technologies are used to process data security, so that the level of information security is increased, and data exchange, storage and sharing can be reliably performed, which provide a strong guarantee for the security of data. Data encryption technology is mainly to encrypts data, and converts plaintext data into hard-to-recognize ciphertext through password protection and technical support, thereby realizing information security protection. Use different ciphertext protection for the key. When the ciphertext is opened, the ciphertext is restored by the key, so that the ciphertext can be presented to the user in a plaintext manner. This is the way the data is decrypted.

\subsection{Protect the firewall of the computers}

With the rapid development of computer networks, computer network security has become a major security risk in people's minds. Firewall technology is an upgrade of the computer network, and there are firewall technologies in the computer network system. This technology is mainly used in home networks, public networks, and specific networks. The development in these networks has led to the widespread application and promotion of firewall technology. Intelligent firewalls have also been promoted in computer networks. In the context of big data network environment, the application of intelligent firewall technology can prevent virus intrusion and propagation, prevent hacker attacks, and protect customer information security. When setting up a firewall for network security in an enterprise, the main way is to restrict the public service from entering the firewall, thereby achieving the purpose of protecting network information security. Firewall technology is mainly applied and filtering. And the way of filtering is static and dynamic. The firewall technology is simple to operate and has high transparency. It can realize network information security protection without changing the original network.

\subsection{Authentication technology}

While protecting the information security of the computer, the encryption technology is used as possible to protect the security of the information, so that the information is guaranteed to be secure. But this information security guarantee has some risks. Therefore, in the process of information protection for computers, information authentication and identity authentication technologies are also an indispensable technology in information security management. Authentication technology mainly includes functions such as digital summarization, digital signature, digital certificate and digital envelope. In the specific application of information security, the main function of the authentication technology is to authenticate and identify the real identity of the user who uses the computer, so that the information is not arbitrarily falsified, deleted and forged, etc., to ensure the security of the information.

\section{Conclusion}

In the era of information explosion, computer technology has been widely used in various fields, and the problems along with the computer information security have followed. Therefore, it is very necessary to strengthen computer information security management. Technical researchers need to innovate in information security technology and put forward corresponding preventive measures to ensure people's network security. 


\section{References}

[1] Dedong Zhang, Yongsheng Zhu, Qun Si. Information security analysis in big data environment[J]. Railway Computer Application, 2015(2):76-78.

[2] Xiaowei Chai. Computer network information security in the era of big data[J]. Computers And Internet, 2016, 42(17):52-53.

[3] Weibing Ding. Research on computer network information security in the era of big data[J]. China Venture Capital, 2016(34).

[4] Ye Yuan. Analysis of computer information security technology in big data environment[J]. Digital Technology and Application, 2017(1):212-213. 